\title{
Damage-free transfer mechanics of 2-dimensional materials: competition between adhesion instability and tensile strain
}

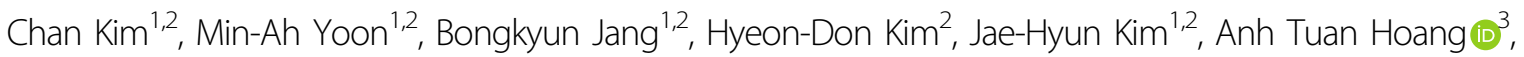 \\ Jong-Hyun Ahn $\mathbb{B}^{3}$, Hyun-June Jung ${ }^{4}$, Hak-Joo Lee ${ }^{4}$ and Kwang-Seop Kim $\mathbb{D}^{1,2}$
}

\begin{abstract}
The transfer of two-dimensional (2D) materials is crucial to the realization of 2D material-based devices for practical applications. The thinness of 2D materials renders them prone to mechanical damage during the transfer process and to degradation of their superior electrical and mechanical properties. Herein, the mechanisms involved in the damage of chemical vapor deposition-grown graphene (Gr) and $\mathrm{MoS}_{2}$ are investigated during a roll-based transfer process. We identify two different damage mechanisms, i.e., instability-induced damage and tensile strain-induced damage. The two mechanisms compete, depending on the thickness of the transfer medium, and induce dissimilar damage. By minimizing these two mechanisms, we realize and demonstrate the damage-free transfer of 2D materials. The sheet resistance and mobility of transferred $\mathrm{Gr}$ are $235 \pm 29 \Omega \mathrm{sq}^{-1}$ and $2250 \mathrm{~cm}^{2} \mathrm{~V}^{-1} \mathrm{~s}^{-1}$, respectively, with no microscopic cracks or tear-out damage. We observe instability-induced damage to be ubiquitous in monolayer MoS $_{2}$, thin metals, and thin oxide films. By understanding the instability-induced damage mechanism, a broad range of 2D materials and thin films can be transferred without mechanical damage. Damage-free transfer will contribute to the high-yield fabrication of 2D material-based electronic devices.
\end{abstract}

\section{Introduction}

The transfer of two-dimensional (2D) materials from their growth substrates onto a target substrate is one of the most important step for fabricating $2 \mathrm{D}$ heterostructures $^{1,2}$ and $2 \mathrm{D}$ material-based devices for practical applications $^{3-5}$. 2D materials such as graphene $(\mathrm{Gr})^{6-8}$, $\mathrm{MoS}_{2}{ }^{9,10}$, and h-BN ${ }^{11-13}$ can be synthesized with high quality on a large scale but are prone to damage when transferred. Transferring large-area 2D materials without any damage is essential for preserving their superior electrical and mechanical properties on a target substrate $^{14,15}$.

\footnotetext{
Correspondence: Kwang-Seop Kim (kskim@kimm.re.kr)

'University of Science \& Technology (UST), Nanomechatronics, 217 Gajeong-ro, Yuseong-gu, Daejeon 34113, Republic of Korea

${ }^{2}$ Nano-Convergence Mechanical Systems Research Division, Korea Institute of Machinery \& Materials (KIMM), 156 Gajeongbuk-ro, Yuseong-gu, Daejeon 34103, Republic of Korea

Full list of author information is available at the end of the article
}

The dry transfer method using a transfer film (TF) is preferred for the large-area transfer of 2D materials because it is readily scalable and compatible with a rollbased continuous transfer process. In 2010, large-area (30-inch) Gr was transferred onto a flexible target substrate using thermal release tape (TRT) as the $\mathrm{TF}^{16}$. The first practical application of large-area Gr transfer was for a touch panel, but TRT residues and cracks in the transferred $\mathrm{Gr}$ were problematic. To overcome these drawbacks, a two-layer TF consisting of a hard supporting film and a thin compliant layer in contact with $\mathrm{Gr}$ via dispersive adhesion was suggested ${ }^{17,18}$. The compliant layer provided conformal contact with the 2D materials, and the supporting layer helped the compliant layer endure the significant deformation that was applied via contact pressure during the roll-based transfer process. The TF was further improved by applying a pressuresensitive adhesive film (PSAF) as the compliant layer ${ }^{19}$. 
The liquid-like PSAF improved the wetting of $\mathrm{Gr}$ on the target substrate and had the lowest surface energy, leading to the decreased formation of ripples and cracks. Some roll-based transfer techniques have also been proposed for the reutilization of metal catalysts; these approaches include electrochemical ${ }^{20-23}$ and direct ${ }^{24-28}$ transfer. In these transfer methods, various polymer films that are a few tens of microns thick, such as silicone, ethylene-vinyl acetate (EVA) copolymer, and polyvinyl alcohol (PVA), are used as the compliant layer. However, the Gr transferred by these methods is still structurally damaged and shows degraded electrical properties.

The compliant layer of a TF can significantly mitigate damage to $2 \mathrm{D}$ materials when the layer is sufficiently soft for conformal contact ${ }^{29}$. Notably, the contact and adhesion forces that occur during the transfer process can lead to the deformation of the compliant layer by a strain that is much larger than the failure strain of the $2 \mathrm{D}$ material ${ }^{30}$. Additionally, the compliant layer should have sufficient adhesion to the 2D material to reduce damage during metal catalyst etching and TF lamination. When a 2D material is transferred onto a target substrate, the adhesion should be weaker than that between the 2D material and the target substrate. Most studies to date have focused on increasing compliance to mitigate damage ${ }^{29}$ and on reducing the surface energy ${ }^{18,19}$ or interfacial energy $^{23,31,32}$ of the compliant layer. However, there are few reports concerning the deformation of the compliant layer that leads to mechanical damage of the 2D material.

Here, we investigate the damage generation mechanisms of 2D materials, such as chemical vapor deposition (CVD)-grown monolayer $\mathrm{Gr}$ and monolayer $\mathrm{MoS}_{2}$, during a roll-based dry transfer process. Contrary to the common belief that damage derives mainly from the interfacial energy difference between $\mathrm{Gr}$ and the contacting substrate, we find that the damage is significantly affected by the thickness-dependent deformation behavior of the compliant layer contacting the 2D material. Depending on the thickness of the compliant layer, two different damage mechanisms are found to be involved in damage generation: instability-induced damage and tensile straininduced damage. In the case of $\mathrm{Gr}$ transfer, when the optimal 100- $\mu \mathrm{m}$-thick polydimethylsiloxane (PDMS) layer is used as the compliant layer, the transferred $\mathrm{Gr}$ has the lowest average sheet resistance of $235 \pm 29 \Omega \mathrm{sq}^{-1}$ for an area of $6 \times 5 \mathrm{~cm}^{2}$ and the highest mobility of $2250 \mathrm{~cm}^{2}$ $\mathrm{V}^{-1} \mathrm{~s}^{-1}$. We confirm that the instability-induced damage mechanism is also applicable to the transfer of monolayer $\mathrm{MoS}_{2}$ and various thin metal and oxide films. These results show that by optimizing the thickness of the compliant layer, damage to the $2 \mathrm{D}$ materials is significantly reduced, and the original electrical properties of the transferred 2D materials are retained. The proposed transfer mechanics are applicable to the transfer of a broad range of 2D materials and provide guidance concerning the damage-free transfer of large-area, highquality $2 \mathrm{D}$ materials.

\section{Materials and methods \\ Sample preparations}

Monolayer $\mathrm{Gr}$ was synthesized on a $35-\mu \mathrm{m}$-thick $\mathrm{Cu}$ foil (JX Nippon Mining and Metals Corp., Japan) using the thermal CVD process ${ }^{33}$. Monolayer $\mathrm{MoS}_{2}$ was grown on a 4-inch-diameter wafer with a 300-nm-thick surfacefunctionalized $\mathrm{SiO}_{2} / \mathrm{Si}$ substrate in a 4.3 inch (inner diameter) hot-wall quartz tube MOCVD system ${ }^{34}$. For the fabrication of the TF, PDMS (Sylgard 184; Dow Corning) and a PET film were used as the compliant layer and supporting film, respectively. Before coating with PDMS, a plasma surface treatment (CUTE plasma system; Femto Science, South Korea) was performed on the PET film to enhance the adhesion between the PDMS and PET film. PDMS was prepared by mixing liquid prepolymer (Sylgard 184 A; Dow Corning, USA) and curing agent (Sylgard 184B; Dow Corning, USA). The mixture was coated as 25-, 50-, 100-, 200-, and 400- $\mu \mathrm{m}$-thick layers onto the PET film. To prepare the $\mathrm{Gr} /$ transfer film $(\mathrm{Gr} / \mathrm{TF})$, TF was laminated onto $\mathrm{Gr}$ grown on a $\mathrm{Cu}$ foil using a homebuilt roll-to-plate (R2P) transfer machine ${ }^{35,36}$. The $\mathrm{Cu}$ foil was etched with $0.1 \mathrm{M}$ ammonium persulfate (APS) solution or a $0.1 \mathrm{M}$ APS solution containing $5 \mathrm{mM}$ imidazole and $50 \mathrm{mM}$ sulfuric acid (IM-APS) ${ }^{33}$. After etching, the $\mathrm{TF} / \mathrm{Gr}$ film was laminated on the $\mathrm{SiO}_{2} / \mathrm{Si}$ substrate under a contact load of $2 \mathrm{~N} / \mathrm{mm}$ and a lamination speed of $0.5 \mathrm{~mm} / \mathrm{s}$ using the R2P transfer machine. Prior to the lamination process, various surface treatments were performed on the $\mathrm{SiO}_{2} / \mathrm{Si}$ substrate. A plasma surface treatment (CUTE plasma system; Femto Science, South Korea) was used to increase the surface energy of the $\mathrm{SiO}_{2} / \mathrm{Si}$ substrate. An amorphous fluoropolymer (Teflon AF 1601; Chemours, USA) was diluted with Fluorinert FC-770 (Sigma-Aldrich, USA) to $0.006 \mathrm{wt} \%$, and the Teflon mixture was coated on the $\mathrm{SiO}_{2} / \mathrm{Si}$ substrate to reduce its surface energy. Then, the substrate was baked in an oven at $160^{\circ} \mathrm{C}$ for $10 \mathrm{~min}$ to remove residual solvent.

\section{Transfer process}

The TF was peeled off in two ways. Crack propagation between the $\mathrm{Gr}$ and PDMS layer in real time was observed using optical microscopy (OM), in which the TF was slowly peeled off by slightly lifting one end of the TF. During the peeling process, crack propagation was observed and recorded by $\mathrm{OM}$ and a charge-coupled device (CCD) camera at a sampling rate of 100 frames/s. For roll-based large-area transfer, the TF was peeled off using the R2P system with precise active load control. In the peeling-off process, the contact load and the peeling- 
off velocity were $0.2 \mathrm{~N} / \mathrm{mm}$ and $0.1 \mathrm{~mm} / \mathrm{s}$, respectively. The radius of the roller in the R2P system was $75 \mathrm{~mm}$. Before transferring $\mathrm{Gr}$, the $\mathrm{SiO}_{2} / \mathrm{Si}$ substrate was treated with plasma for $30 \mathrm{~s}$ (CUTE plasma system; Femto Science, South Korea) to increase the surface energy of the substrate. For the transfer of $\mathrm{MoS}_{2}$, a TF/MoS$/ \mathrm{SiO}_{2} / \mathrm{Si}$ stack was placed in a deionized (DI) water bath for waterassisted separation. Then, one end of the TF was peeled upward, and crack propagation was observed and recorded by OM and a CCD camera at a sampling rate of 100 frames/s.

\section{Characterization}

The adhesion properties of the TF were estimated using a home-built adhesion tester ${ }^{37}$. A laser-quality fused silica planoconvex lens (PLCX-8.0-25.8-UV; CVI Melles Griot, USA) was used as the counterpart material for the adhesion test. In the test, the contact load was $10 \mathrm{mN}$, and the dwell time was $100 \mathrm{~s}$. The TF was separated from the lens at a prescribed separation velocity that ranged from 2 to $500 \mu \mathrm{m} / \mathrm{s}$. The contact force and contact area were measured in the test, and the adhesion strength was calculated by dividing the pull-off force by the critical contact area. Raman spectra were obtained using a Raman spectrometer with a 514-nm laser as the excitation source (inVia Raman microscope; Renishaw, UK). The beam size of the laser was $2 \mu \mathrm{m}$ using a $50 \times$ objective lens. Images of the transferred $\mathrm{Gr}$ were obtained by scanning electron microscopy (SEM, JSM-7610 FPlus field-emission SEM; JEOL, Japan) operating at less than $1 \mathrm{kV}$ to suppress charging. The topography of the samples was investigated using atomic force microscopy (AFM, XE-100; Park Systems, South Korea). A Si probe (NCHR, Park Systems, South Korea) was used to measure the topography over an area of $5 \times 5 \mu^{2}$ at a scan rate of $0.5 \mathrm{~Hz}$. The sheet resistance of $\mathrm{Gr}$ was measured in two ways: before the transfer process, the sheet resistance of $\mathrm{Gr}$ on a TF was measured using a noncontact resistivity tester (EC-80P; Napson, Japan) with a noncontact probe (NC-5CL; Napson, Japan). The probe contacted the back side of the $\mathrm{Gr}$ / TF (i.e., not on the Gr side) to prevent mechanical damage to the Gr surface; after the transfer process, the sheet resistance of the $\mathrm{Gr}$ transferred onto the $\mathrm{SiO}_{2} / \mathrm{Si}$ substrate was measured using a four-point probe nanovoltmeter (Model 6221; Keithley, USA). The Hall mobility of the transferred $\mathrm{Gr}$ was measured using a Hall effect measurement system (HMS-5300; Ecopia, South Korea) at $10 \mathrm{~mA}$.

\section{Finite-element (FE) analysis}

The roll-transfer process of $\mathrm{Gr}$ was modeled for finiteelement analysis in the 2D plane strain condition using ABAQUS commercial code. The steel roll and the flat substrate were considered rigid bodies without any deformation, and the blanket was firmly attached without slippage. Additionally, we assumed that the $50-\mathrm{mm}$-wide TF (PET/PDMS) was tightly bonded to the surface of the blanket. The contact condition between the adhesive layer and substrate was deemed frictionless. When the $2 \mathrm{~N} / \mathrm{mm}$ load from the transfer process was applied, we calculated the strain distribution in the deformable blanket and the TF. Young's modulus and Poisson's ratio of the materials used in the model are listed in Table S1.

\section{Results and discussion}

To investigate the damage mechanism of 2D materials, the transfer of CVD-grown monolayer $\mathrm{Gr}$ onto an $\mathrm{SiO}_{2} / \mathrm{Si}$ wafer was observed in real time using $\mathrm{OM}$ and a $\mathrm{CCD}$ camera system. Figure 1a illustrates the preparation of a TF sample and a $\mathrm{TF} / \mathrm{Gr} /$ substrate $\left(\mathrm{SiO}_{2} / \mathrm{Si}\right)$ stack sample. The compliant layer of the TF was formed by coating a thin PDMS layer onto a polyethylene terephthalate (PET) film. A home-built R2P transfer machine with active load control was used for all lamination processes ${ }^{35,36}$. The TF/Gr/ substrate stack was placed on the microscope stage, and the TF was slowly peeled from one end of the TF (Fig. 1b). Figure 1c shows real-time OM images of the propagation of the interfacial crack front as the TF was peeled from the substrate. The shape of the interfacial crack front varied greatly and depended on the thickness of the PDMS layer $(25,100$, or $400 \mu \mathrm{m})$ (Movie S1, Supporting Information); additionally, the PDMS surface energy was constant (Fig. S1, Supporting Information). After the complete removal of the TF, the transferred $\mathrm{Gr}$ on the $\mathrm{SiO}_{2} / \mathrm{Si}$ wafer was observed again using OM and SEM. Figure 1d, e shows that the shape of the damage depended on the thickness of the PDMS layer. Notably, for the transfer using a $100-\mu \mathrm{m}$ thick PDMS layer, no structural damage was observed over an area of a few tens of square centimeters. In contrast, when the thickness of the PDMS layer was 25 or $400 \mu \mathrm{m}$, the transferred Gr was severely cracked and torn. Raman spectroscopy and AFM confirmed that there was no $\mathrm{Gr}$ in the damaged areas (Fig. S2, Supporting Information). Using TFs with different PDMS layer thicknesses enabled large-area $\mathrm{Gr}$ to be transferred onto the $\mathrm{SiO}_{2} / \mathrm{Si}$ substrate under well-controlled transfer conditions using the R2P transfer machine (Fig. 1f). Figure 1g shows a histogram of the sheet resistance of the transferred $\mathrm{Gr}$ according to the thickness of the PDMS layer. The average sheet resistance of $\mathrm{Gr}$ was the lowest when the thickness of the PDMS layer was $100 \mu \mathrm{m}$, at $235 \pm 29 \Omega \mathrm{sq}^{-1}$; the inset of Fig. $1 \mathrm{~g}$ shows that the sheet resistance was quite uniform over an area of $6 \times 5 \mathrm{~cm}^{2}$ (Fig. S3b, e, Supporting Information). On the other hand, when the thickness of the PDMS layer was 25 or $400 \mu \mathrm{m}$, the average sheet resistance of $\mathrm{Gr}$ was higher at $1130 \pm 42$ and $563 \pm 202 \Omega \mathrm{sq}^{-1}$, respectively. The distribution of the sheet resistance was severely nonuniform (Fig. S3a, c, Supporting Information), even when the same 

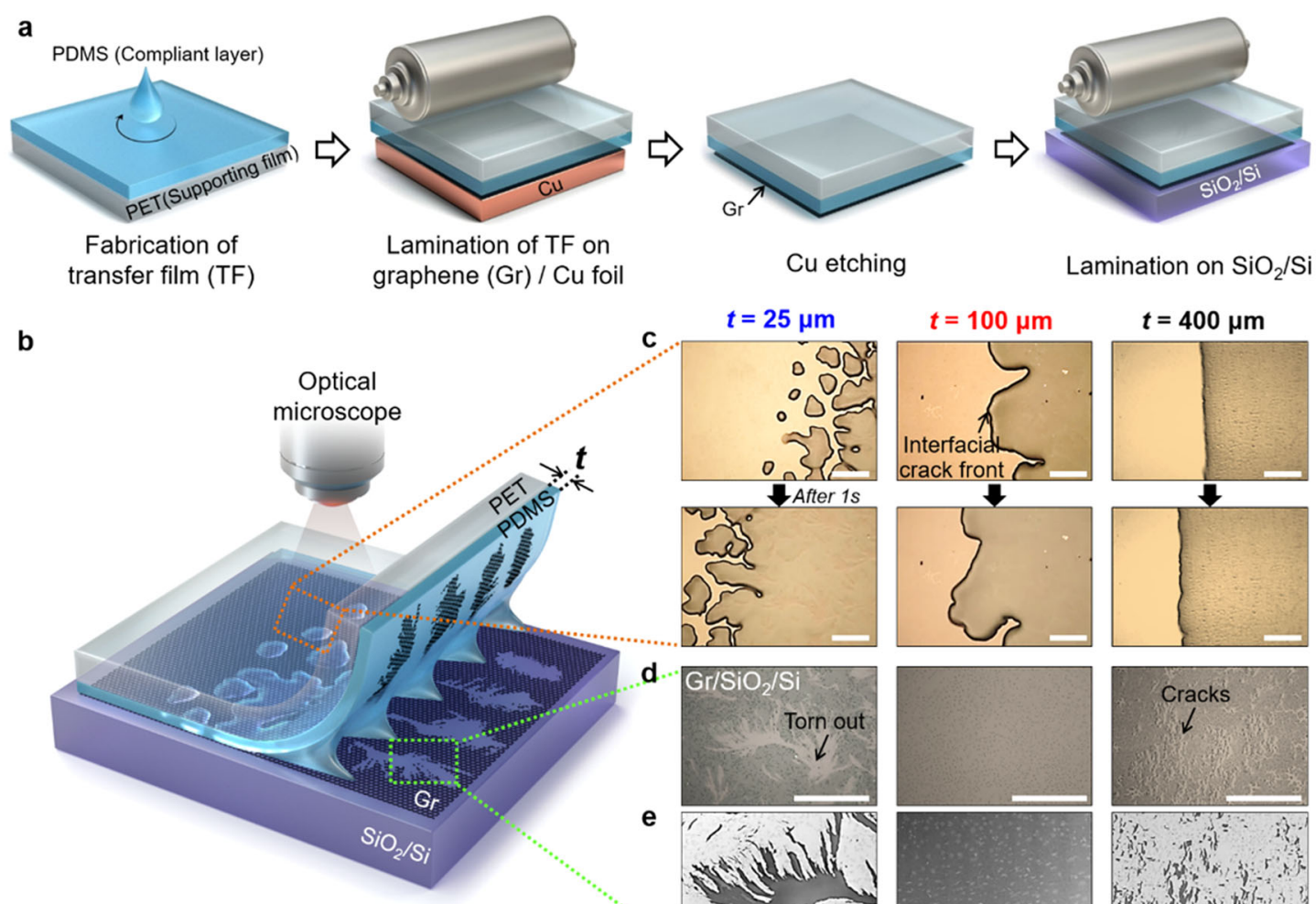

Lamination on $\mathrm{SiO}_{2} / \mathrm{Si}$

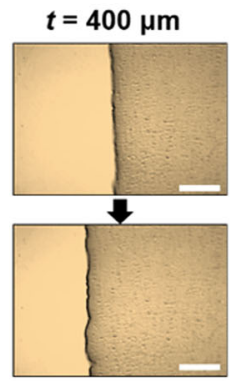

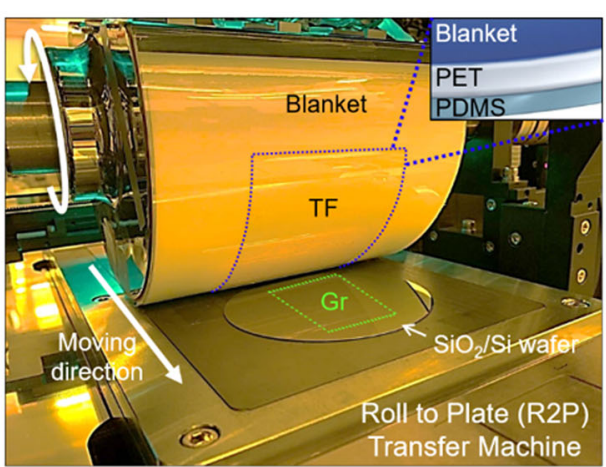

d. $\mathrm{Gr} / \mathrm{SiO}_{2} / \mathrm{Si}$
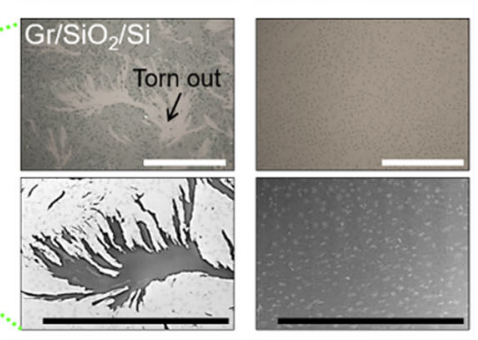

$\mathrm{g}_{25}$
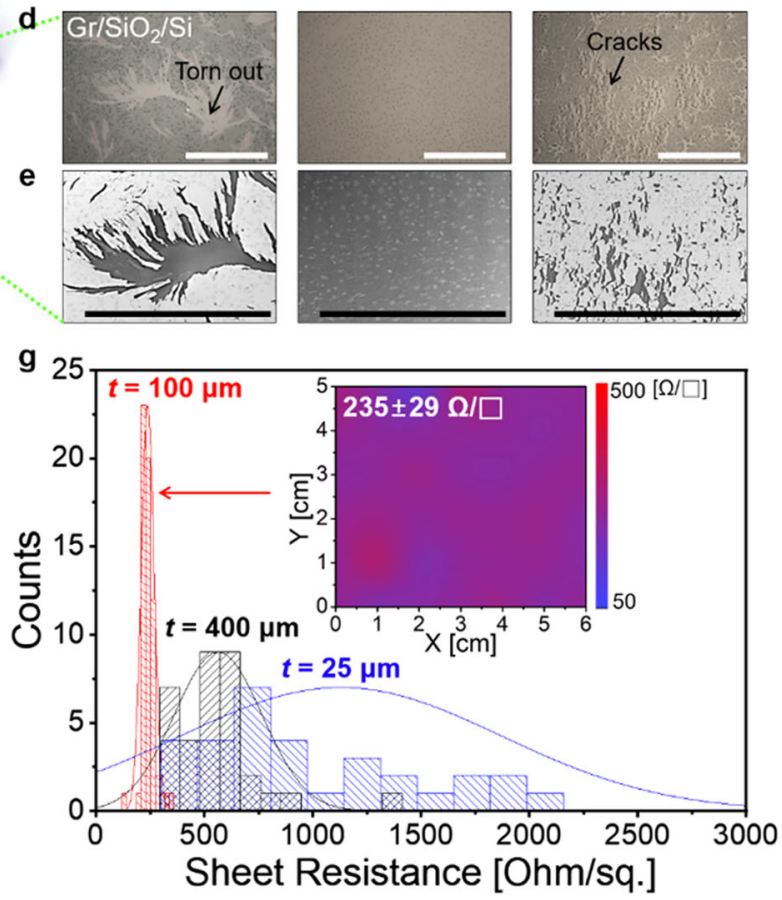

Fig. 1 Mechanical damage of the transferred graphene (Gr) induced by the thickness-dependent deformation of the PDMS layer.

a Schematic illustration showing the preparation of the transfer film (PET/PDMS)/Gr/substrate $\left(\mathrm{SiO}_{2} / \mathrm{Si}\right)$ stack. Samples were prepared with different thicknesses of the PDMS layer in the TF. The lamination process was performed using a roll-to-plate (R2P) transfer machine with active load control. b Schematic illustration showing the transfer test used to observe the interfacial crack front in real time using optical microscopy (OM). c In situ OM images of the interfacial crack front during the peeling process for TFs having different PDMS layer thicknesses $(t=25,100$, and $400 \mu m)$. The interfacial crack front propagated from right to left within $1 \mathrm{~s}$. After completely peeling off the TF, the transferred Gr on the substrate was observed using $\mathbf{d} \mathrm{OM}$ and e scanning electron microscopy (SEM). The optical microscopy and SEM images showed the difference in structural damage to the transferred Gr according to the thickness of the PDMS layer. In the case of the TF with a 100- $\mu$ m-thick PDMS layer, structural damage was hardly visible in the transferred $\mathrm{Gr}$. The scale bars in $\mathbf{c}$, d and e represent $200 \mu \mathrm{m}$. $\mathbf{f}$ Photograph of the R2P transfer machine used to transfer large-area Gr onto the $\mathrm{SiO}_{2} / \mathrm{Si}$ substrate using the TFs. $\mathbf{g}$ Histogram of the sheet resistance of the transferred Gr. The histogram showed that the sheet resistance of the Gr transferred using the TF containing a 100- $\mu \mathrm{m}$-thick PDMS layer had the lowest value and was uniform over an area of $6 \times 5 \mathrm{~cm}^{2}$ (inset).

transfer conditions were used. This higher sheet resistance was likely responsible for the structural damage generated during the roll-transfer process. Scanning electron microscopy images of Gr transferred by the R2P machine show the different damage shapes that depended on the PDMS thickness (Fig. S3d-f, Supporting Information). 

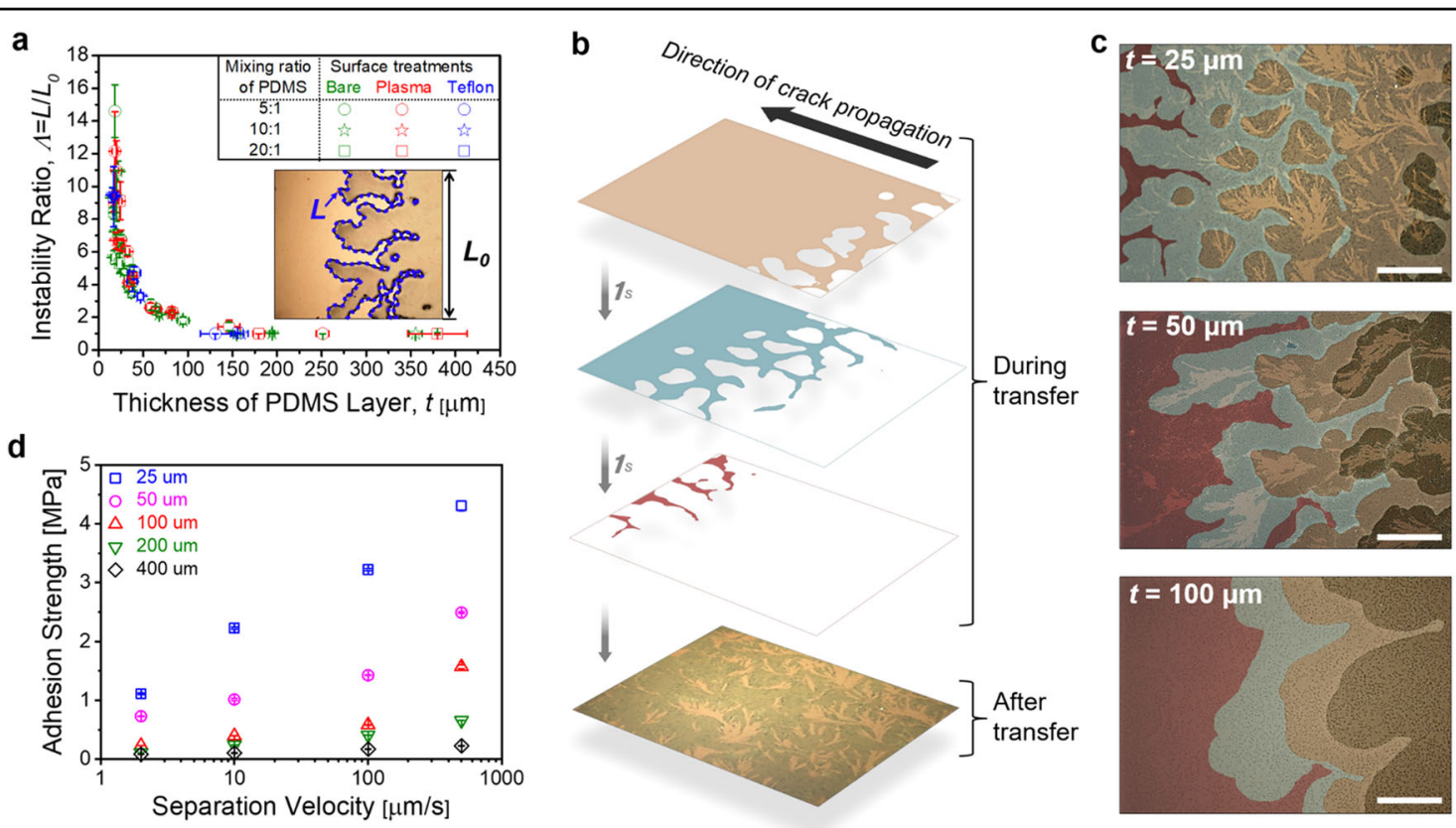

Fig. 2 Adhesion-induced instability of the PDMS layer and the resultant structural damage to the transferred Gr. a Instability ratio as a function of the PDMS layer thickness, PDMS mixing ratio, and $\mathrm{SiO}_{2} / \mathrm{Si}$ substrate surface treatment. The instability ratio rapidly increased for thicknesses less than $100 \mu \mathrm{m}$. The instability ratio was defined as the overall length $L$ (blue dotted line) of the interfacial crack front divided by the reference length $L_{0}$ in the images captured during the transfer process. $\mathbf{b}$ Superimposed images captured during the transfer and the images after the transfer at the same location of the sample. In the images captured during transfer, the contact areas of the PDMS layer with the substrate are colored pink, cyan, and red for the time step of $1 \mathrm{~s}$ so that the changes in the instability shape can be observed in respect to time. c Superimposed images according to the PDMS layer thickness. For the transfer film with 25- or 50- $\mu$ m-thick PDMS layers, the propagation shape of the instability with time coincided well with the structural damage to the transferred $\mathrm{Gr}$; the scale bar represents $200 \mu \mathrm{m}$. $\mathbf{d}$ Rate-dependent adhesion characteristics of the PDMS layer for different PDMS layer thicknesses. The adhesion strength of the PDMS layer increased with an increasing separation velocity, and the increasing rate of adhesion strength rapidly increased with a decreasing PDMS layer thickness.

The trend in shape in relation to thickness resembles that shown in Fig. 1d, e. These results confirm that the thickness of the PDMS layer used as the compliant layer was one of the main causes of damage in both the laboratoryscale manual transfer and the roll-based large-area transfer of Gr. Thus, careful attention should be given to the thickness of the compliant layer to avoid mechanical damage to the transferred Gr. Therefore, the quality of transferred $\mathrm{Gr}$ could be significantly improved merely by adjusting the compliant layer thickness. The following paragraphs detail the effect of the compliant layer thickness on the damage generation mechanism.

As the thickness of the PDMS layer decreased below $100 \mu \mathrm{m}$, the shape of the interfacial crack front during TF peeling changed into a complex shape resembling fingers and ripples. This behavior is characteristic of the adhesion-induced instability of a confined thin film ${ }^{38}$. When a thin compliant layer with a shear modulus of less than $10 \mathrm{MPa}$ is peeled off, thinner layers are more inclined to deform laterally than thicker layers; thus promoting instability to release the elastic strain energy stored in the film ${ }^{39,40}$. The shape of the instability is predominantly influenced by the layer thickness, rather than the material properties and the surface energy of the contacting materials ${ }^{40-42}$. To quantify the instability, we defined the instability ratio $(\Lambda)$ as the overall length $(L)$ of the interfacial crack front divided by the reference length $\left(L_{0}\right)$ $\left(\Lambda=L / L_{0}\right.$, inset of Fig. 2a). For example, the instability ratio was 1 when there was no instability in the interfacial crack front. Figure 2a shows that the instability ratio increased rapidly with a decreasing PDMS thickness, especially below a thickness of approximately $50 \mu \mathrm{m}$. The elastic modulus of the PDMS significantly changed with the mixing ratio, while the surface energy of the PDMS layer was unchanged (Fig. S4, Supporting Information). The surface treatments greatly changed the surface energy of the $\mathrm{SiO}_{2} / \mathrm{Si}$ substrates. Our findings revealed that the instability ratio was affected mainly by the PDMS thickness, while the elastic modulus of the PDMS and the surface energies of the substrate rarely affected the instability ratio.

To investigate the relationship between instability and damage to the transferred $\mathrm{Gr}$, we superimposed the OM images of the interfacial crack front captured every $1 \mathrm{~s}$ during the transfer process onto the image of the transferred Gr observed at the same location after completely 
peeling off the TF (Fig. 2b). Figure 2c shows the resultant superimposed images for the PDMS layers with different thicknesses and reveals that the shape of the damage generated on the transferred $\mathrm{Gr}$ coincides well with the propagation shape of the instability in the PDMS layers for layer thicknesses of 25 and $50 \mu \mathrm{m}$. Regarding these layers, the interfacial crack front at the end of the fingers propagated much faster (Fig. S5, Supporting Information), despite the slow peeling speed of the TF; therefore, the damage spread along the propagation direction of the instability. However, when the thickness was $100 \mu \mathrm{m}$, some instability occurred, but not severely, and no structural damage was evident on the transferred $\mathrm{Gr}$. Figure $2 \mathrm{~d}$ shows the measured adhesion strength of the PDMS layers as a function of separation velocity. As the separation velocity increased, so did the adhesion strength, indicating that the adhesion characteristics of the PDMS layer were rate-dependent ${ }^{25,37,43,44}$. The increase in adhesion strength was more substantial for PDMS layers thinner than $100 \mu \mathrm{m}$. Due to the instability, the PDMS layer rapidly separated from the substrate at the end of the fingers. The adhesion between the layer and Gr was significantly higher due to the rate-dependent adhesion characteristics of the layer. The increased adhesion tore $\mathrm{Gr}$ from the substrate, corresponding to the propagation shape of the instability. In addition to thin PDMS layers, some commercially available TFs with 25$\mu$ m-thick silicone or $80-\mu$ m-thick EVA layers as the compliant layer can also cause instability-induced damage to transferred Gr (Fig. S6, Supporting Information). Therefore, for a PDMS layer thinner than $100 \mu \mathrm{m}$, the damage to the transferred $\mathrm{Gr}$ was mainly caused by instability. In addition, the 100- $\mu$ m-thick PDMS layer left little residue on the transferred $\mathrm{Gr}$ compared to the commercial TFs (Fig. S7, Supporting Information).

When the thickness of the PDMS layer exceeded $150 \mu \mathrm{m}$, the instability ratio was almost 1 (Fig. 2a), indicating the absence of instability. However, crack-like damage, which was not caused by instability, was observed on the transferred $\mathrm{Gr}$ when the thickness of the PDMS layer was $400 \mu \mathrm{m}$ (Fig. 1d, e and Fig. S3f, Supporting Information). To investigate the damage mechanism for the thick PDMS layers, finite-element models (FEMs) were developed to simulate the mechanical contact that occurred during the roll-transfer process for PDMS thicknesses of 100, 200, and $400 \mu \mathrm{m}$. Figure 3a illustrates a

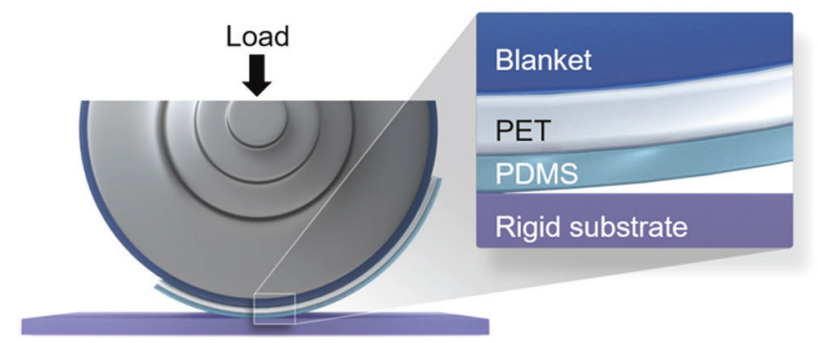

C

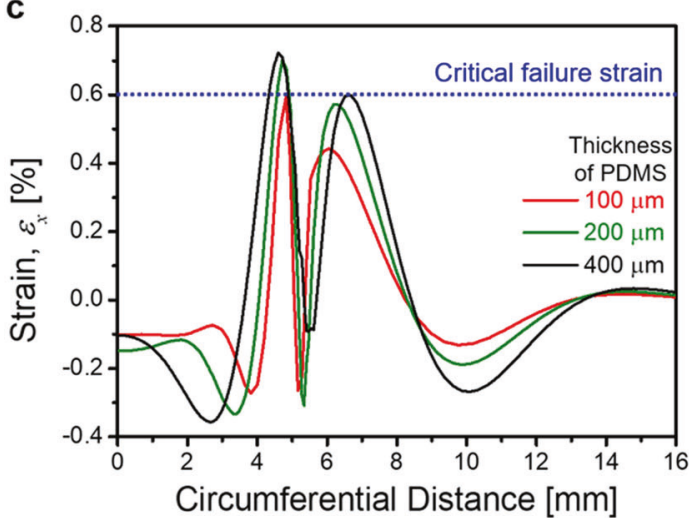

b

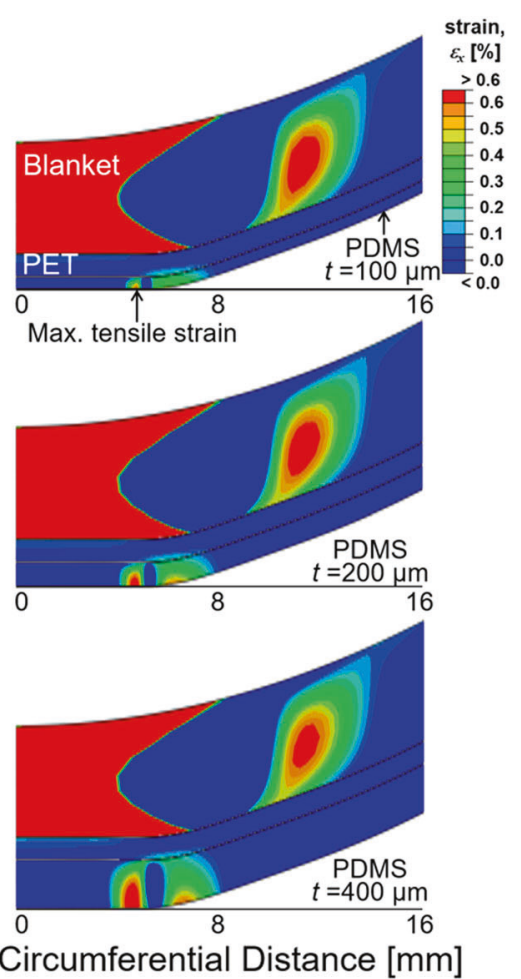

Fig. 3 Finite-element analysis of the roll-to-plate (R2P) transfer process and simulated strain distribution according to PDMS layer thickness. a Schematic illustration showing the finite-element model; the transfer film (PET/PDMS) was fixed on a soft blanket, and the PDMS layer came into contact with the rigid substrate under a contact load of $2 \mathrm{~N} / \mathrm{mm}$. $\mathbf{b}$ Contours of the in-plane strain $\varepsilon_{x}$ along the circumferential direction of the roll according to the PDMS layer thickness. The PDMS layer experienced maximum tensile strain near the end of the contact area. $\mathbf{c}$ Profiles of the in-plane strain $\varepsilon_{x}$ in the PDMS layer surface as a function of thickness. The critical failure strain of Gr/PET was approximately $0.6 \%{ }^{45,46}$. 
the FEM used to calculate strain distributions. A 1-mmthick soft blanket was wrapped around a rigid stainlesssteel roll with a radius of $75 \mathrm{~mm}$, and the TF (PET/PDMS) was fixed on the blanket without slippage. Since the blanket had a lower elastic modulus than that of the PDMS, it was possible to avoid nonuniform contacts caused by mechanical misalignment in the R2P machine. The analysis was performed for PDMS layer thicknesses of 100, 200, and $400 \mu \mathrm{m}$. The blanket/PET/PDMS layer stack deformed when in contact with a rigid substrate when the applied contact load per unit width was $2 \mathrm{~N} /$ $\mathrm{mm}$; this parameter was equal to the experimental condition of R2P lamination. The mechanical properties, layer thicknesses, boundary conditions, and calculation details are described in the Methods section and Table S1 of the Supporting Information. Figure $3 \mathrm{~b}$ shows the contour of the in-plane strain $\varepsilon_{x}$ along the circumferential direction of the roll according to the thickness of the PDMS layer. The soft, thick blanket was largely deformed along the circumferential direction by the contact pressure, while the PET supporting film with a relatively large elastic modulus was hardly deformed, and the strain of the blanket was not transferred to the PDMS layer. However, the thin PDMS layer deformed significantly near the contact area between the layer and substrate because of the deformed geometries in the layered structure. At the contact edge, the radius of curvature of the PDMS surface abruptly converted from a finite to an infinite value, which resulted in tensile deformation in the thickness direction. Hence, compressive deformation in the circumferential direction at the contact edge occurred due to Poisson's effect, which resulted in tensile deformation in the adjacent region of the contact edge. This phenomenon was also observed in the transfer process when a roll coated with a thin and deformable film was used ${ }^{35,36}$. In this study, the larger area over which the sudden fluctuation of $\varepsilon_{x}$ in the PDMS layer increased with an increasing thickness was due to the geometric effect. Figure 3c shows the variation in strain $\varepsilon_{x}$ at the PDMS surface as a function of the circumferential distance from the center. The $100-\mu \mathrm{m}$-thick PDMS layer experienced a maximum tensile strain of $\sim 0.6 \%$ near the end of the contact area. As the thickness increased to above $200 \mu \mathrm{m}$, the maximum tensile strain increased by more than $0.7 \%$, which exceeded the critical failure strain of the CVD-monolayer $\mathrm{Gr}$ transferred onto the PET film. Previous research established that $\mathrm{Gr}$ started to crack along the direction perpendicular to the applied tensile strain, and the resistance of the Gr/PET increased accordingly when the tensile strain exceeded $0.6 \%{ }^{45,46}$. The FEM analysis indicated that when the thickness of the PDMS layer exceeded $100 \mu \mathrm{m}$, the maximum tensile strain in the PDMS layer caused by the contact pressure exceeded the failure strain of the CVD Gr, causing the Gr to crack. This mechanism explained why crack-like damage was observed mainly for the Gr transferred via the 400- $\mu \mathrm{m}$-thick PDMS layer and why the damage had a different shape than the instabilityinduced damage on the $\mathrm{Gr}$ transferred via the $25-\mu \mathrm{m}$ thick PDMS layer (Fig. 1c, d).

As explained above, the damage mechanism depended on the thickness of the PDMS layer in contact with Gr. The electrical properties of the transferred $\mathrm{Gr}$ also changed with the PDMS layer thickness. Figure 4a shows the change in the sheet resistance of Gr before and after the R2P transfer process. As the PDMS layer thickness decreased below $80 \mu \mathrm{m}$, the relative sheet resistance increased significantly due to increased instability-induced damage, regardless of the mixing ratio of the PDMS. As the thickness of the PDMS layer increased above $150 \mu \mathrm{m}$, the relative sheet resistance gradually increased due to increased tensile strain-induced damage. Therefore, there was an optimal layer thickness that minimized both damage mechanisms. When the thickness of the PDMS layer made at a mixing ratio of 10:1 ranged from 90 to $150 \mu \mathrm{m}$, the change in the sheet resistance of $\mathrm{Gr}$ before and after the R2P transfer process was less than $10 \%$. The same trend was noted in the mobility measurement (Fig. 4b). Similar to the observed changes in sheet resistance, the mobility also increased rapidly with an increasing PDMS thickness, with a maximum mobility of $2250 \mathrm{~cm}^{2}$ $\mathrm{V}^{-1} \mathrm{~s}^{-1}$ recorded at a PDMS thickness of $100 \mu \mathrm{m}$. Then, the mobility gradually decreased with an increasing thickness. The $2 \mathrm{D}$ and $\mathrm{D}$ peaks of the transferred graphene without mechanical damage were comparable to those of the as-grown graphene in the mapping area. (Fig. S8, Supporting Information). Figure $4 \mathrm{c}$ summarizes the damage mechanisms that depended on the compliant layer thickness. In the case of a compliant layer that was thin enough to cause adhesion instability, the propagation velocity of the interfacial crack front increased locally at the end of the fingers. In this region, the adhesion between the layer and Gr was enhanced by the rate-dependent adhesion characteristics of the layer, allowing the $\mathrm{Gr}$ to adhere to the layer and not transfer onto the substrate. As the thickness of the compliant layer increased, the adhesion instability disappeared, but the compliance of the layer increased in proportion to the thickness. Therefore, when the tensile strain of the layer along the circumferential direction of the roll due to contact pressure exceeded the failure strain of $\mathrm{Gr}$ at the edge of the contacted area, $\mathrm{Gr}$ cracked perpendicularly to the rolling direction. A TF for damage-free transfer should be carefully designed because the mechanical properties and thickness of the materials comprising the TF can alter the tensile strain of the compliant layer.

The instability-induced damage mechanism is applicable to $\mathrm{MoS}_{2}$ transfer as well as to $\mathrm{Gr}$ transfer. $\mathrm{MoS}_{2}$ was grown on a $\mathrm{SiO}_{2} / \mathrm{Si}$ wafer using the metal-organic chemical vapor 

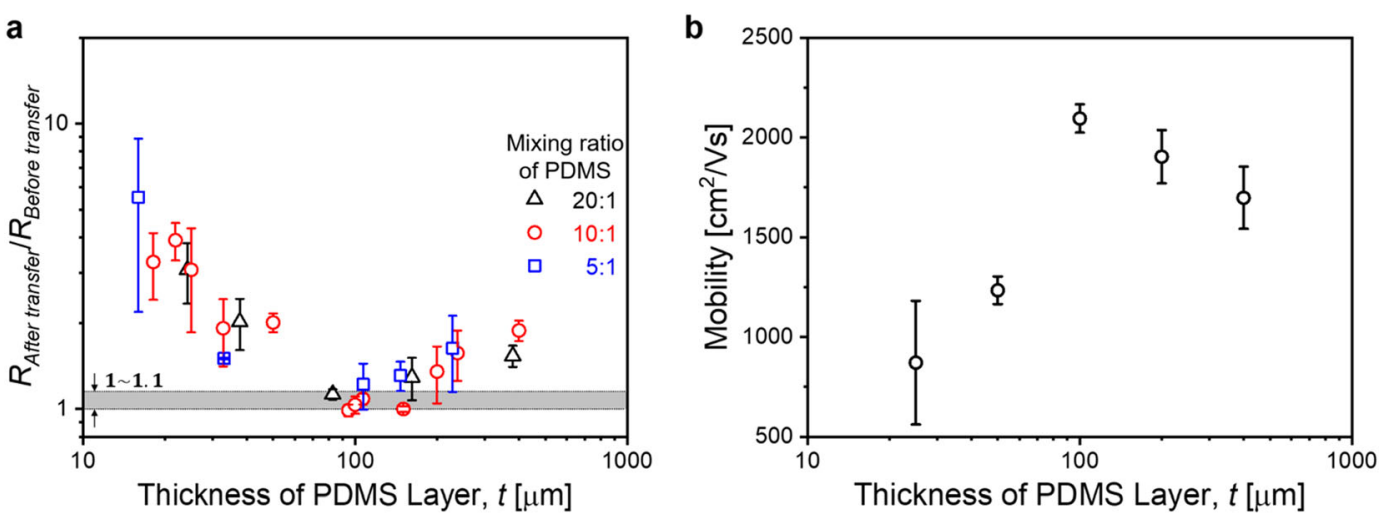

C
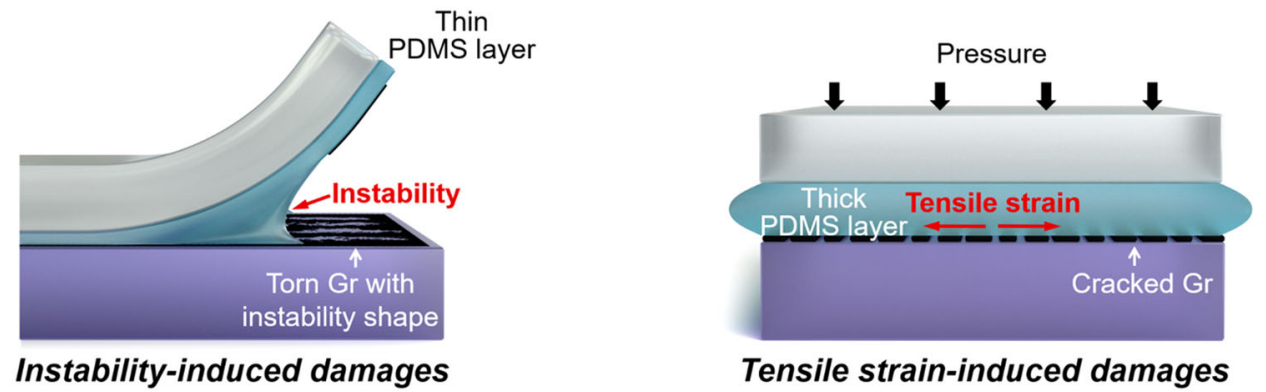

Fig. 4 Electrical properties of the transferred Gr as a function of the PDMS layer thickness and schematics showing the damage generation mechanisms according to the layer thickness. a Sheet resistance changes of the transferred Gr before and after the transfer process as a function of the PDMS layer thickness. When the thickness of the PDMS layer was approximately $100 \mu \mathrm{m}$, the change in sheet resistance was minimal due to the reduced structural damage irrespective of the PDMS mixing ratio. $\mathbf{b}$ Mobility of the transferred Gr as a function of the PDMS layer thickness after the transfer process. The PDMS mixing ratio was 10:1. c Schematic illustration showing the structural damage mechanism occurring during the transfer process and the role of the PDMS layer thickness.

deposition (MOCVD) process $^{34}$ and confirmed to be a monolayer (Fig. S9, Supporting Information). Figure 5a shows the transfer process of monolayer $\mathrm{MoS}_{2}$ from the growth substrate to the TF using water-assisted separation. Transfer films with different PDMS thicknesses were laminated onto the $\mathrm{MoS}_{2}$ samples using the R2P transfer machine. The resulting $\mathrm{TF} / \mathrm{MoS}_{2}$ samples were then placed in a deionized (DI) water bath to enable water penetration at the $\mathrm{MoS}_{2} /$ substrate interface ${ }^{34,47,48}$. When the end of the TF was peeled upward, the $\mathrm{MoS}_{2}$ separated from the substrate, and interfacial crack propagation was observed in real time by OM (Fig. 5b and Movie S2, Supporting Information). In the case of the TF with the $25-\mu \mathrm{m}$-thick PDMS layer, severe adhesion instability occurred at the interfacial crack front during the separation process. In contrast, in the case of the TF with the $100-\mu \mathrm{m}$-thick PDMS layer, such severe instability did not occur. The surfaces of the TF and the substrate were observed after complete separation. Figure $5 \mathrm{c}$ shows that when the thickness of the PDMS layer was $25 \mu \mathrm{m}, \mathrm{MoS}_{2}$ was partly transferred onto the PDMS surface in the shape of the propagated instability. The rest of the $\mathrm{MoS}_{2}$ remained exactly on the substrate but in the reverse shape
(Fig. 5d). When the PDMS layer was $100 \mu \mathrm{m}$ thick, instability-induced damage did not occur during separation, and $\mathrm{MoS}_{2}$ was transferred well from the substrate to the PDMS layer. Figure 5e, f shows AFM images of the $\mathrm{MoS}_{2}$ that remained on the substrate and the Raman mapping of the $A_{1 g}$ peak for the specified area. When the $\mathrm{MoS}_{2} /$ substrate was peeled off by a TF with a $100-\mu \mathrm{m}$ thick PDMS layer, the $\mathrm{MoS}_{2}$ was clearly removed in most areas, and the $\mathrm{MoS}_{2}$ remaining on the substrate was observed only at the edge of the contacted area (Fig. 5e). In contrast, when $\mathrm{MoS}_{2}$ was transferred by the $25-\mu \mathrm{m}$-thick PDMS layer, $\mathrm{MoS}_{2}$ was peeled off with a sharp saw-tooth shape at the edge of the delaminated area along the direction of the propagated instability (Fig. 5f). The Raman mapping data of the $\mathrm{A}_{1 \mathrm{~g}}$ peak at the specified areas revealed that $\mathrm{MoS}_{2}$ remained uniformly on the substrate after delamination. In addition, it was confirmed by photoluminescence $(\mathrm{PL})$ mapping that the crystal quality of the transferred $\mathrm{MoS}_{2}$ was not degraded when $\mathrm{MoS}_{2}$ was transferred by the transfer film with $100 \mu \mathrm{m}$-thick PDMS (Fig. S10, Supporting Information).

As noted above, instability-induced tear-out damage and tensile-strain-induced crack-like damage of the transferred 


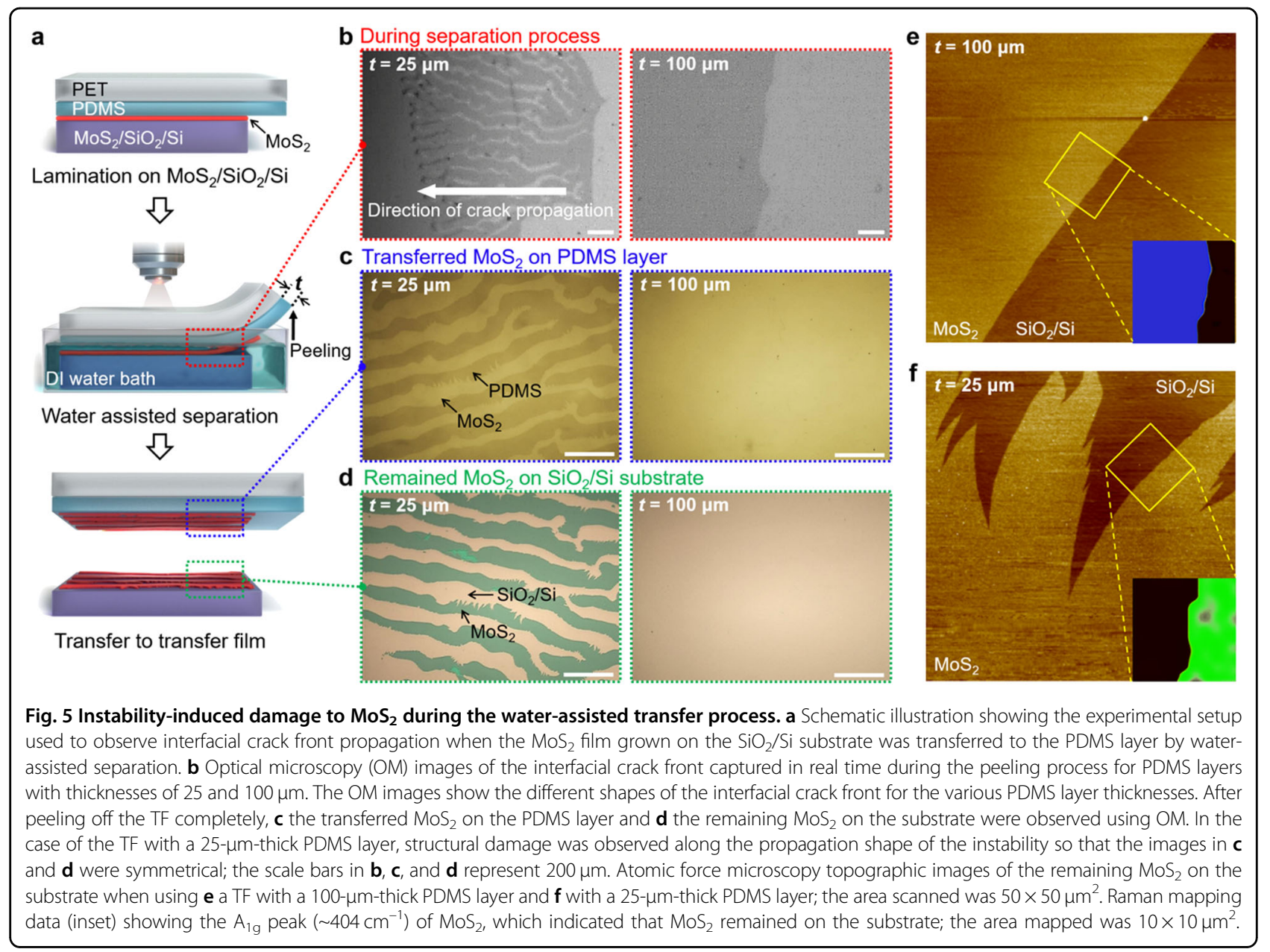

2D materials were closely connected to the thicknessdependent deformation behavior of the compliant layers. In addition, the thickness of the compliant layers affected the conformal contact between the layer and 2D materials because the compliance of the layers decreased with decreasing layer thickness ${ }^{29,49}$. However, most research concerning the transfer of $2 \mathrm{D}$ materials using a TF with a thin compliant layer, such as silicone ${ }^{17-19}$, EVA $^{20-24,26}$, $\mathrm{PVA}^{28}$, and $\mathrm{PDMS}^{6,44}$, did not give attention to the compliant layer thickness or its deformation behavior under transfer conditions. Consequently, the quality of transferred 2D materials often varies between research groups because of differences in the compliant layer thickness, even when almost the same transfer method is used. The damage mechanism according to the thickness-dependent deformation behavior of the compliant layer was not limited to the transfer of 2D materials and could be extended to the transfer of various thin films, such as metal and oxide layers. Transferring several tens of nanometer-thick thin films of $\mathrm{Au}$ and Al-doped $\mathrm{ZnO}$ (AZO) using a TF with a 25- $\mu \mathrm{m}$-thick PDMS layer led to instability-induced damage (Fig. S11, Supporting Information). In addition to the compliant layer thickness, the contact load applied to the TF/2D materials/substrate stack was another critical factor that should be carefully controlled to achieve conformal contact between 2D materials and the substrate and reduce the tensile strain-induced damage of $2 \mathrm{D}$ materials in the lamination and transfer processes (Fig. S12, Supporting Information). In most previous studies, large-area Gr was transferred using a simple laminator system ${ }^{16,18,26}$, which was typically operated with spring-loaded rollers without active load control. When using the system, the contact pressure and resultant tensile strain in the compliant layer could vary greatly depending on the total thickness of the stacked sample. Therefore, to improve the transfer yield, careful attention must be given to the thickness of the compliant layer in contact with the 2D material and the contact load applied to the stacked sample during the entire transfer process.

\section{Conclusions}

In conclusion, we found that the thickness-dependent deformation behavior of a thin compliant layer in a TF played a crucial role in controlling the damage to $2 \mathrm{D}$ 
materials during the transfer process. The two damage mechanisms of instability-induced damage and tensile strain-induced damage had a tradeoff relationship with respect to the thickness of the compliant layer. This result meant that there was an optimal thickness that minimized the overall damage. By optimizing the thickness of the compliant layer, the damage-free transfer of $2 \mathrm{D}$ materials could be achieved. Herein, when we used the optimal 100$\mu \mathrm{m}$-thick PDMS layer, the transferred Gr had the best quality and was free of cracks and other damage. The sheet resistance and mobility of the transferred $\mathrm{Gr}$ were $235 \pm$ $29 \Omega \mathrm{sq}^{-1}$ and $2250 \mathrm{~cm}^{2} \mathrm{~V}^{-1} \mathrm{~s}^{-1}$, respectively. No degradation was observed in the sheet resistance before and after the transfer process, indicating that the transferred Gr was damage-free. We observed instability-induced damage ubiquitously in monolayer $\mathrm{MoS}_{2}$, thin metal films, and thin oxide films. Understanding the instability-induced damage mechanism enables the damage-free transfer of a broad range of $2 \mathrm{D}$ materials and thin films onto arbitrary substrates. These findings will facilitate the high-yield fabrication of 2D material-based electronic devices.

\section{Acknowledgements}

This work was supported by the Center for Advanced Meta-Materials (CAMM), which is funded by the Ministry of Science, ICT, and Future Planning as a Global Frontier Project (CAMM No. 2014063701, 2014063700), and by the Korea Institute of Energy Technology Evaluation and Planning (KETEP, No. 20183010014310), which is funded by the Ministry of Trade, Industry, and Energy (MOTIE) of the Republic of Korea. This work was also supported by an internal research program of the Korean Institute of Machinery and Materials (NK224D).

\section{Author details \\ 'University of Science \& Technology (UST), Nanomechatronics, 217 Gajeong-ro, Yuseong-gu, Daejeon 34113, Republic of Korea. ${ }^{2}$ Nano-Convergence \\ Mechanical Systems Research Division, Korea Institute of Machinery \& Materials (KIMM), 156 Gajeongbuk-ro, Yuseong-gu, Daejeon 34103, Republic of Korea. ${ }^{3}$ School of Electrical and Electronic Engineering, Yonsei University, 50 Yonsei- ro, Seodaemun-gu, Seoul 03722, Republic of Korea. ${ }^{4}$ Center for Advanced Meta-Materials (CAMM), 156 Gajeongbuk-ro, Yuseong-gu, Daejeon 34103, Republic of Korea}

\section{Conflict of interest}

The authors declare no competing interests.

\section{Publisher's note}

Springer Nature remains neutral with regard to jurisdictional claims in published maps and institutional affiliations.

Supplementary information The online version contains supplementary material available at https://doi.org/10.1038/s41427-021-00311-1.

Received: 11 December 2020 Revised: 18 March 2021 Accepted: 6 April 2021.

Published online: 14 May 2021

\footnotetext{
References

1. Novoselov, K. S., Mishchenko, A., Carvalho, A. \& Castro Neto, A. H. 2D materials and van der Waals heterostructures. Science 353, aac9439 (2016).
}

2. Kang, K. et al. Layer-by-layer assembly of two-dimensional materials into wafer-scale heterostructures. Nature 550, 229-233 (2017).

3. Liu, Y., Huang, Y. \& Duan, X. Van der Waals integration before and beyond two-dimensional materials. Nature 567, 323-333 (2019).

4. Bae, S. H. et al. Integration of bulk materials with two-dimensional materials for physical coupling and applications. Nat. Mater. 18, 550-560 (2019).

5. Akinwande, D. et al. Graphene and two-dimensional materials for silicon technology. Nature 573, 507-518 (2019).

6. Lee, Y. et al. Wafer-scale synthesis and transfer of graphene films. Nano Lett. 10, 490-493 (2010)

7. Polsen, E. S., McNerny, D. Q., Viswanath, B., Pattinson, S. W. \& John Hart, A. High-speed roll-to-roll manufacturing of graphene using a concentric tube CVD reactor. Sci. Rep. 5, 10257 (2015).

8. Deng, B., Liu, Z. \& Peng, H. Toward mass production of CVD graphene films. Adv. Mater. 31, 1800996 (2019).

9. Lee, Y. H. et al. Synthesis of large-area $\mathrm{MoS}_{2}$ atomic layers with chemical vapor deposition. Adv. Mater. 24, 2320-2325 (2012).

10. Yang, P. et al. Batch production of 6-inch uniform monolayer molybdenum disulfide catalyzed by sodium in glass. Nat. Commun. 9, 979 (2018).

11. Lee, J. S. et al. Wafer-scale single-crystal hexagonal boron nitride film via selfcollimated grain formation. Science 362, 817-821 (2018).

12. Wang, L. et al. Epitaxial growth of a 100-square-centimetre single-crystal hexagonal boron nitride monolayer on copper. Nature 570, 91-95 (2019).

13. Chen, T. A. et al. Wafer-scale single-crystal hexagonal boron nitride monolayers on $\mathrm{Cu}$ (111). Nature 579, 219-223 (2020).

14. Chen, Y., Gong, X. L. \& Gai, J. G. Progress and challenges in transfer of largearea graphene films. Adv. Sci. 3, 1500343 (2016).

15. Ma, L., Ren, W. \& Cheng, H. Transfer methods of graphene from metal substrates: a review.Small Methods 3, 1900049 (2019).

16. Bae, S. et al. Roll-to-roll production of 30-inch graphene films for transparent electrodes. Nat. Nanotechnol. 5, 574-578 (2010).

17. Chen, X. D. et al. High-quality and efficient transfer of large-area graphene films onto different substrates. Carbon 56, 271-278 (2013).

18. Choi, T. et al. Roll-to-roll continuous patterning and transfer of graphene via dispersive adhesion. Nanoscale 7, 7138-7142 (2015).

19. Kim, S. J. et al. Ultraclean patterned transfer of single-layer graphene by recyclable pressure sensitive adhesive films. Nano Lett. 15, 3236-3240 (2015).

20. Wang, Y. et al. Electrochemical delamination of CVD-grown graphene film: toward the recyclable use of copper catalyst. ACS Nano 5, 9927-9933 (2011).

21. Hempel, M. et al. Repeated roll-to-roll transfer of two-dimensional materials by electrochemical delamination. Nanoscale 10, 5522-5531 (2018)

22. Chandrashekar, B. N. et al. A universal stamping method of graphene transfer for conducting flexible and transparent polymers. Sci. Rep. 9, 3999 (2019).

23. Tavakoli, M. M. et al. Synergistic roll-to-roll transfer and doping of CVDgraphene using parylene for ambient-stable and ultra-lightweight photovoltaics. Adv. Funct. Mater. 30, 2001924 (2020).

24. Juang, Z. Y. et al. Graphene synthesis by chemical vapor deposition and transfer by a roll-to-roll process. Carbon 48, 3169-3174 (2010).

25. $\mathrm{Na}$, S. R. et al. Selective mechanical transfer of graphene from seed copper foil using rate effects. ACS Nano 9, 1325-1335 (2015).

26. Chandrashekar, B. N. et al. Roll-to-roll green transfer of CVD graphene onto plastic for a transparent and flexible triboelectric nanogenerator. Adv. Mater. 27, 5210-5216 (2015).

27. Xin, H., Zhao, Q., Chen, D. \& Li, W. Roll-to-roll mechanical peeling for dry transfer of chemical vapor deposition graphene. J. Micro Nano-Manuf. 6, 031004 (2018).

28. Shivayogimath, A. et al. Do-it-yourself transfer of large-area graphene using an office laminator and water. Chem. Mater. 31, 2328-2336 (2019).

29. Hong, J. et al. A rational strategy for graphene transfer on substrates with rough features. Adv. Mater. 28, 2382-2392 (2016).

30. Jang, B. et al. Damage mitigation in roll-to-roll transfer of CVD-graphene to flexible substrates. 2D Mater. 4, 1-10 (2017).

31. Song, J. et al. A general method for transferring graphene onto soft surfaces. Nat. Nanotechnol. 8, 356-362 (2013).

32. Zhang, Z. et al. Rosin-enabled ultraclean and damage-free transfer of graphene for large-area flexible organic light-emitting diodes. Nat. Commun. 8, 14560 (2017).

33. Jo, K. et al. One-step etching, doping, and adhesion-control process for graphene electrodes. Carbon 82, 168-175 (2015). 
34. Shinde, S. M. et al. Surface-functionalization-mediated direct transfer of molybdenum disulfide for large-area flexible devices. Adv. Funct. Mater. 28 1706231 (2018).

35. Sharma, B. K. et al. Load-controlled roll transfer of oxide transistors for stretchable electronics. Adv. Funct. Mater. 23, 2024-2032 (2013).

36. Choi, M. et al. Stretchable active matrix inorganic light-emitting diode display enabled by overlay-aligned roll-transfer printing. Adv. Funct. Mater. 27, 1606005 (2017)

37. Kim, C. et al. Ultimate control of rate-dependent adhesion for reversible transfer process via a thin elastomeric layer. ACS Appl. Mater. Interfaces 9 12886-12892 (2017)

38. Ghatak, A., Chaudhury, M., Shenoy, V. \& Sharma, A. Meniscus instability in a thin elastic film. Phys. Rev. Lett. 85, 4329-4332 (2000).

39. Shenoy, V. \& Sharma, A. Pattern formation in a thin solid film with interactions. Phys. Rev. Lett. 86, 119-122 (2001).

40. Ghatak, A. \& Chaudhury, M. K. Adhesion-induced instability patterns in thin confined elastic film. Langmuir 19, 2621-2631 (2003)

41. Biggins, J. S., Saintyves, B., Wei, Z., Bouchaud, E. \& Mahadevan, L. Digital instability of a confined elastic meniscus. Proc. Natl Acad. Sci. USA 110 12545-12548 (2013)
42. Chaudhury, M. K., Chakrabarti, A. \& Ghatak, A. Adhesion-induced instabilities and pattern formation in thin films of elastomers and gels. Eur. Phys. J. E $\mathbf{3 8}, 82$ (2015).

43. Jang, B. et al. Rate-dependent adhesion between a spherical PDMS stamp and silicon substrate for a transfer-assembly process. J. Adhes. 87, 744-754 (2011).

44. Seo, J. et al. Direct graphene transfer and its application to transfer printing using mechanically controlled, large area graphene/copper freestanding layer. Adv. Funct. Mater. 28, 1707102 (2018).

45. Won, S. et al. Double-layer CVD graphene as stretchable transparent electrodes. Nanoscale 6, 6057-6064 (2014).

46. Lee, J.-H. et al. Fracture mechanism and electromechanical behavior of chemical vapor deposited graphene on flexible substrate under tension. Carbon 118, 475-484 (2017).

47. Lai, S., Jeon, J., Song, Y.-J. \& Lee, S. Water-penetration-assisted mechanical transfer of large-scale molybdenum disulfide onto arbitrary substrates. RSC Adv. 6, 57497-57501 (2016).

48. Phan, H. D. et al. Ultraclean and direct transfer of a wafer-scale MoS 2 thin film onto a plastic substrate. Adv. Mater. 29, 1603928 (2017).

49. Shull, K. R. Contact mechanics and the adhesion of soft solids. Mater. Sci. Eng. R. Rep. 36, 1-45 (2002). 